\title{
Successful robot-assisted surgery for treating endometrial cancer affecting bicornuate-bicollis or didelphic uterus
}

\author{
Annamaria Maglione, Sergio Montanaro, Francesco Petruzzelli \\ Obstetrics and Gynecology Unit, IRCCS “Casa Sollievo della Sofferenza”, San Giovanni Rotondo, Italy \\ Email: annamaria.maglione@libero.it
}

Received 21 April 2013; revised 23 May 2013; accepted 1 June 2013

Copyright (C) 2013 Annamaria Maglione et al. This is an open access article distributed under the Creative Commons Attribution License, which permits unrestricted use, distribution, and reproduction in any medium, provided the original work is properly cited.

\begin{abstract}
Uterine anomalies are mainly associated with improper development of Mullerian ducts. To our knowledge, reports describing the use of a da Vinci Robot System for treating an endometrial cancer in a woman with an abnormal uterus are lacking. Here, we reported the description of two extremely obese women, affected by endometrial cancer and presenting bicornuate-bicollis and didelphic uterus have been treated by Robot System-assisted surgery. No operative and postoperative complications were recorded. Both women are disease-free to follow-up. A robot-assisted surgery could represent an effective treatment option for complex hysterectomies and in complicated patients, as those showing an extreme obesity.
\end{abstract}

Keywords: Uterine Malformations; Endometrial Cancer; Robot-Assisted-Surgery

\section{INTRODUCTION}

Uterine anomalies are mainly associated with improper development of Mullerian ducts [1]. In unselected populations, the prevalence of these anomalies range from $0.16 \%$ to $10 \%$ [2]. However, the number of cases could be underestimated. Women who have genital tract malformations can be asymptomatic thus the diagnosis is delayed and more often is done in occasion of gynecological examinations intended to investigate other pathologies, such as reproductive problems. Bicornuate uterus can originate from an incomplete fusion of Mullerian ducts or from a failed and partial resorption of median septum following fusion process. A systematic review [3] has evidenced that the embriological-clinical classification of female genital tract malformations appears to be the most appropriate. The classification provides six groups of anomalies. The first includes cases of unicornuate uterus with contralateral renal agenesis due to agenesis or hypoplasia of an entire urogenital ridge. Then, we can find uterine duplicities (bicornuate or didelphys uterus) with a blind hemivagina (or unilateral cervicovaginal atresia) and ipsilateral renal agenesis. The third is characterized by anomalies in the Mullerian development processes also included in the classification of the American Fertility Society without other associated anomalies; and also the transverse vaginal septum. The fourth presents accessory uterine masses with an otherwise normal uterus, and other possible gubernaculum dysfunctions. Anomalies of the urogenital sinus and malformative combinations close the list of the categories. The association between endometrial cancer and Mullerian duct anomalies is uncommon [4-6]. Furthermore, anomalies of female genital tract are not supposed to be risk factors associated with the occurrence of cancer affecting this anatomical area. To our knowledge, reports describing a robot-assisted surgery for treating an endometrial cancer in a woman with an abnormal uterus are lacking. Every year about 600,000 hysterectomies are performed in the United States, the majority of them via laparotomy. The introduction of advanced laparoscopic technology made total laparoscopic hysterectomy more feasible. Conventional laparoscopy, however, has its limitations. Limited dexterity, range of movement and 2-dimensional vision make complex surgical tasks difficult [7]. As a result, many physicians are deterred from utilizing a laparoscopic approach to hysterectomy, especially in patients with larger uteri, obesity, and prior surgery. Robotic surgery is the latest innovation in the field of minimally invasive surgery. Robotic surgical systems have been used to perform surgery for endometrial, cervical and ovarian cancer. There is mounting evidence which demonstrates the feasibility and safety of robotic surgery for gynaecological oncology [8]. Robotic surgery represents a minimally invasive treatment option. The da Vinci Surgical System (Intuitive Surgical, Inc., Sunnyvale, CA, USA) (Figure 1) was cleared by the US Food and Drug Administration 


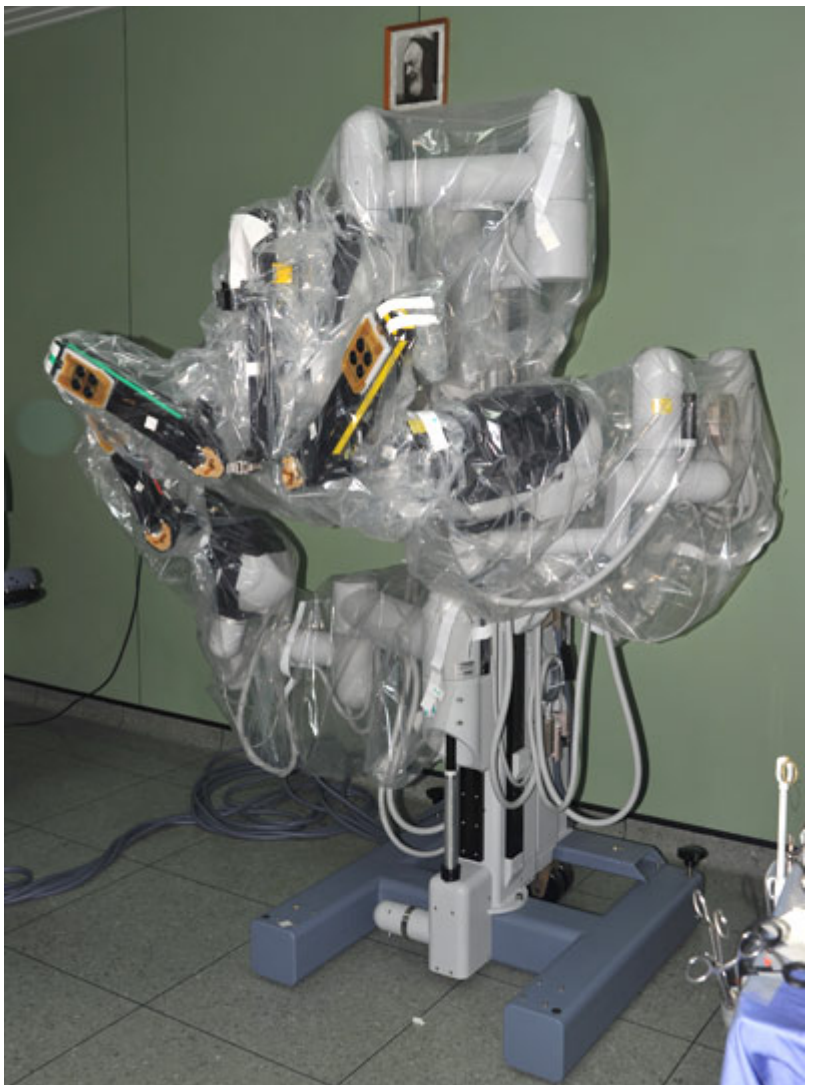

Figure 1. Da Vinci ${ }^{\circledR}$ surgical system.

(FDA) in 2005 for gynecological procedures and rapidly gained acceptance by surgeons as an effective device for performing hysterectomy with staging lymphadenectomy in the management of endometrial cancer [9]. The da Vinci Robot System-assisted surgery provides a highresolution 3D image of the surgical site, and allows surgeons to perform precise movements and to operate with a better dexterity and control.

Currently, there are limited data that comparing outcomes of total laparoscopic to a robotic approach to hysterectomy for the treatment of benign and malignant gynecologic pathology and the studies often fail to control for surgeon and other possible confounding factors [10]. A uterine neoplasm in a woman with malformations of the genital tract could be a diagnostic challenge for gynaecologists. Women needing curettage because of postmenopausal bleeding could represent undiagnosed patients for endometrial cancer whether present a double uterine cavity. Here is the description of two cases of endometrial cancer affecting an abnormal uterus and treated by a Da Vinci Robot System-assisted surgery.

A 65-year-old obese (body mass index 31.3) and nulliparous woman with sterility problems and hypertensive disorders underwent hysteroscopy because of bleeding episodes. She was diagnosed with endometrial adenocarcinoma which localized in the smallest cavity of a bi- cornuate-bicollis uterus. A NMR application, covering pelvis and abdomen, and a trans-vaginal ultrasound have allowed to stage the cancer which was found to be in an early phase. The woman received a hysterectomy, a bilateral annessiectomy and a lymphadenectomy by a da Vinci Robot System-assisted surgery (Figure 2).

The total duration of anesthetic was $41 / 2$ hours. Laparoscopic surgery required 3 hours and $50 \mathrm{~min}$ and the surgeon console time was 3 hours and $15 \mathrm{~min}$. The histological examination has revealed and confirmed the presence of an early stage cancer (p1b G2 N0), associated with a total number of 10 lymph nodes. Blood loss during surgery was equal to $20 \mathrm{ml}$. No operative and postoperative complications were recorded. The closure of vaginal vault was performed via vaginal approaches. Patient was discharged on the third day after surgery. After 19 months follow-up the patient is disease-free.

A 65-year-old (Figure 3) extremely obese (body mass index 67) weighing $155 \mathrm{~kg}$. She was affected by mellitus diabetes and hypertension, and showed an obstetric anamnesis characterised by three caesarean section. During post-menopausal period, she suffered from bleeding episodes. A diagnostic hysteroscopy was performed and revealed the presence of an endometrioid adenocarcinoma localizing in the left cavity of a didelphic uterus (Figure 4) associated with a double vagina.

A NMR application, covering pelvis and abdomen, and a trans-vaginal ultrasound have allowed to stage the cancer which was found to be in an early phase. Because of obesity, patient was not eligible for radiotherapy. Thus, she was a good candidate for a robot-assisted surgery. Adesiolysis was performed because of adhesion formation occurring during caesarean section. The woman received a hysterectomy, a bilateral annessiectomy and a pelvic lymphadenectomy by a da Vinci Robot Systemassisted surgery. The total duration of anesthetic was 5 $1 / 2$ hours. Laparoscopic surgery required 4 hours and 20

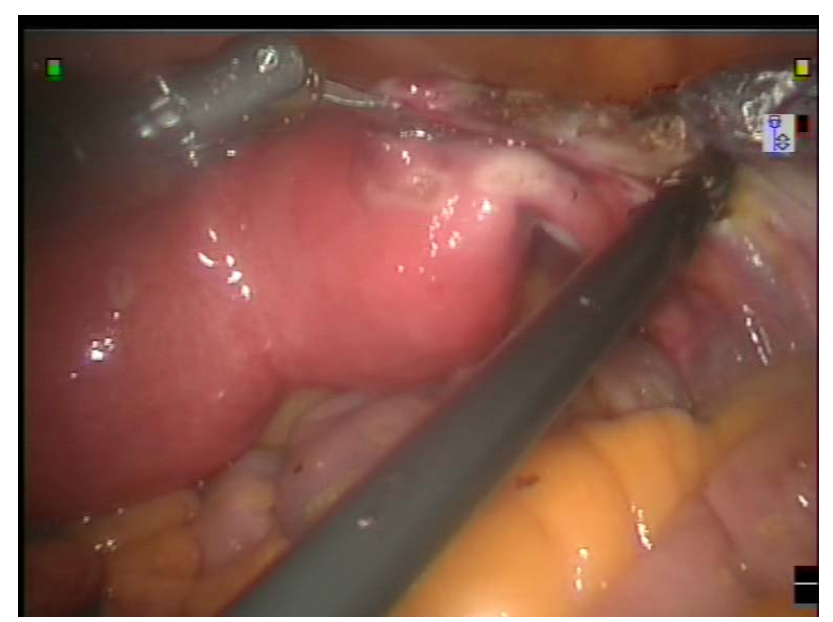

Figure 2. Robot-assisted surgery in Cases \#1. 


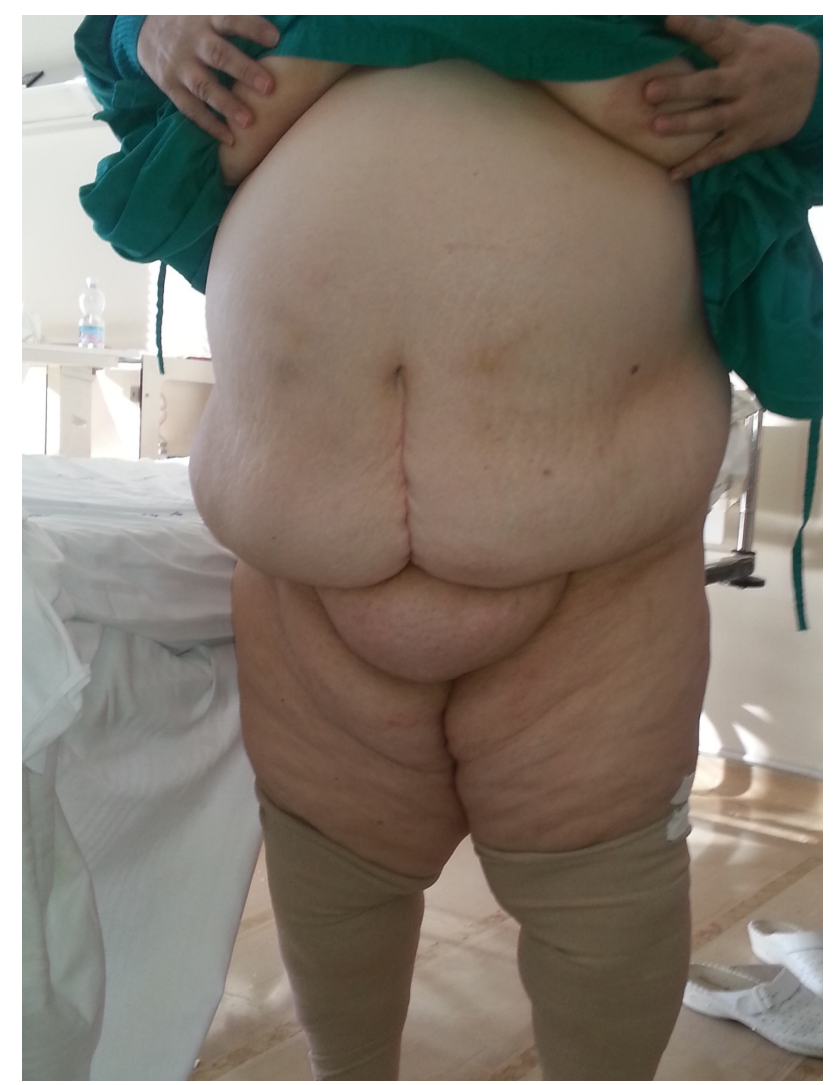

Figure 3. Case \#2.

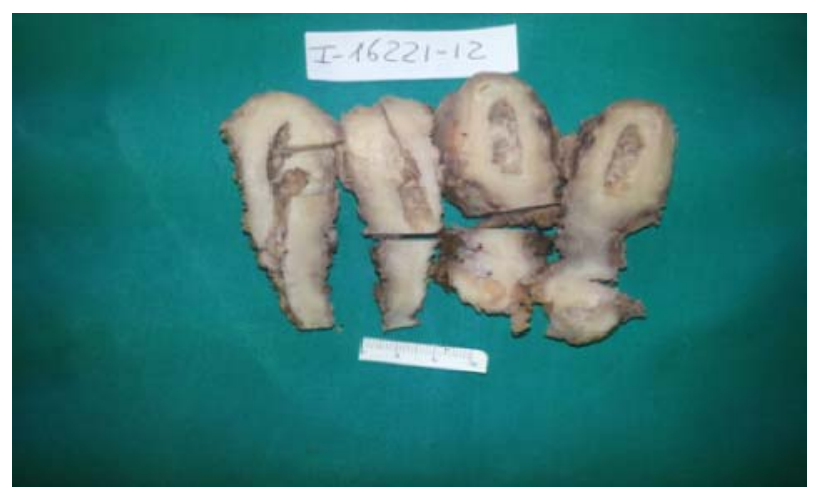

Figure 4. The didelphic uterus of the Case \#2.

min and the surgeon console time was 3 hours and 20 min. The histological examination has confirmed the presence of an early stage cancer (p1b G2 N0). Blood loss during surgery was equal to $30 \mathrm{ml}$. No operative and post-operative complications were recorded. The closure of vaginal vault was performed via robot-assisted technique. Patient was discharged on the third day after surgery.

\section{COMMENT}

To date, the few cases of gynecological neoplasm affecting abnormal uterus have not been treated through a robot-assisted surgery and were not obese patients. A robot-assisted technique could enable the surgeon to perform a more precise and accurate anatomical dissection. The integrity of vessels and nerves appear to be mainly preserved during a robot-assisted surgery. Particularly, a da Vinci Robot System allows seven degrees of freedom, a three-dimensional and intuitive visualization, as well as provides ergonomic comfort. Potential benefits that a robot-assisted surgery could offer include less pain, small incisions, fewer complications, less blood loss, shorter hospital stay, low risk of wound infection and a quicker return to normal activities. Additional medical implications associated with obesity and anatomical peculiarities of uterus of these cases have lead us to perform a robotassisted surgery, due to better operative conditions and minimally invasive features of this surgical techinique. Obesity is associated with a 10-fold increased risk for endometrial cancer and is often mentioned as a "limiting factor” concerning patient selection for either laparoscopic or robotic procedures. Obese patients have an increased risk of laparotomy conversion and less complete lymph nodal dissection [9]. There are only few recent studies comparing robotic with laparoscopic hysterictomy and most of them are retrospective [11]. Additionally, managing obese patients with endometrial cancer through a robot-assisted surgery is still poorly understood because of limitations this innovative procedure has regarding low node counts and significantly high rate of conversion to laparotomy. Other important limitations of robotic surgery exist including the added operative time and cost associated with the robotic procedure [10]. To date, transfusions, hospital stay and complications seem to be improved for robotic-assisted surgery [9]. Robotic surgical systems have high fixed costs, with prices ranging from \$1 million to \$2.5 million for each unit. Surgeons must perform 150 to 250 procedures to become adept in their use. The systems also require costly maintenance and demand the use of additional consumables (single-use robotic appliances). The use of robotic systems may also require more operating time than alternatives. In the case of procedures that had previously been performed as open surgery, however, some of the new costs will be offset by reductions in postoperative hospital costs and by productivity gains if patients recover more rapidly and can return to work and other activities sooner [12]. Thus, a robot-assisted surgery could represent an effective treatment option for complex hysterictomies, such as those received from women with uterine malformations, such as those characterized by a double cavity. Added to this is that benefits associated with a robot-assisted surgery seems to be mainly more important than abdominal and vaginal surgery and traditional laparoscopy when patients who are treated present critical clinical conditions, such as obesity. Fast recovery of 
both patients and excellent clinical outcomes suggest that robot-assisted surgery could represent a valid therapeutic procedure for treating cancer patients with critical uterine malformations and at higher risk of experiencing perioperative and post-operative complications because of the extreme obesity. We reported the description of two complex endometrial cancer cases successfully treated by a robot-assisted surgery. However, more researches are needed to assess clinical and social advantages of this innovative technology in the field of gynecology surgery.

\section{REFERENCES}

[1] Dane, C. (2009) A single horn endometrial carcinoma of a uterus bicornis unicollis. Journal of Gynecologic Oncology, 20, 195-197. doi:10.3802/jgo.2009.20.3.195

[2] Shulman, L.P. (2008) Mullerian anomalies. Clinical Obstetrics and Gynecology, 51, 214-222. doi:10.1097/GRF.0b013e31816feba0

[3] Acien, P. and Acien, M.I. (2011) The history of female genital tract malformation classifications and proposal of an updated system. Human Reproduction Update, 17, 693-705. doi:10.1093/humupd/dmr021

[4] Duncan, A.S. and John, A.H. (1962) Endometrial carcinoma occurring in uterus bicornis. Journal of Obstetrics and Gynaecology of the British Empire, 69, 488-490. doi:10.1111/j.1471-0528.1962.tb01181.X

[5] Tsukahara, Y., Fukamatsu, Y., Tomita, K., Shiozawa, T., Iinuma, H. and Fukuta, T. (1990) Endometrial carcinoma arising from a double uterus. Gynecologic and Obstetric Investigation, 29, 311-312. doi:10.1159/000293344

[6] Kosinski, A. and Dini, M. (1994) Endometrial cancer in a double uterus: A report of two cases. Journal of Reproductive Medicine, 39, 926-927.

[7] Shashoua, A.R., Gill, D. and Locher, S.R. (2009) Robotic-assisted total laparoscopic hysterectomy versus conventional total laparoscopic hysterectomy. Journal of the Society of Laparoendoscopic Surgeons, 13, 364-369.

[8] Lu, D., Liu, Z., Shi, G., Liu, D. and Zhou, X. (2012) Robotic assisted surgery for gynaecological cancer. Cochrane Database of Systematic Reviews, 1, CD008640. doi:10.1002/14651858.CD008978.pub2

[9] Holloway, R.W. and Ahmad, S. (2012) Robotic-assisted surgery in the management of endometrial cancer. Journal of Obstetrics and Gynaecology Research, 38, 1-8. doi:10.1111/j.1447-0756.2011.01744.X

[10] Soto, E., Lo, Y., Friedman, K., Soto, C., Nezhat, F., Chuang, L. and Gretz, H. (2011) Total laparoscopic hysterectomy versus da Vinci robotic hysterectomy: Is using the robot beneficial? Journal of Gynecologic Oncology, 22, 253-259. doi:10.3802/jgo.2011.22.4.253

[11] Sarlos, D. and Kots, L.A. (2011) Robotic versus laparoscopic hysterectomy: A review of recent comparative studies. Current Opinion in Obstetrics and Gynecology, 23, 283-288. doi:10.1097/GCO.0b013e328348a26e

[12] Barbash, G.I. and Glied, S.A. (2010) New technology and health care costs-The case of robot-assisted surgery. New England Journal of Medicine, 363, 701-704. doi:10.1056/NEJMp1006602 